\title{
Harmful alcohol consumption in elite sports players in Ireland
}

\author{
Kieran Murray ${ }^{1}\left({ }^{\circ} \cdot\right.$ Colm Murphy $^{2} \cdot$ Ann Herlihy $^{3} \cdot$ Jack McCaffrey $^{4} \cdot$ Mary Codd $^{5} \cdot$ Frank E. Murray $^{6,7}$
}

Received: 21 May 2021 / Accepted: 13 October 2021 / Published online: 26 October 2021

(c) The Author(s), under exclusive licence to Royal Academy of Medicine in Ireland 2021

\begin{abstract}
Background Sportspeople are more prone to binge drink than their peers.

Aims We aimed to assess alcohol consumption, harms and behaviours in an elite Irish sporting population (Gaelic footballers and hurlers).

Methods An anonymous web-based questionnaire (demographics, alcohol consumption, culture and related harms) was administered to all elite players. The AUDIT-C questionnaire (frequency, quantity of alcohol consumption and frequency of binge drinking) was used to assess for adverse alcohol use. Univariate and multivariate analyses assessed for predictors of adverse alcohol use.

Results 717 players (mean age 24 years) were analysed. The majority of patients were male (75\%), unmarried (93\%) and had completed university (67\%). 96\% were current drinkers. Players consumed more alcohol during the off-season (median 20 versus 8 standard drinks in 28 days) compared to the elite season. Amongst current drinkers, $73 \%$ exhibit adverse alcohol use, $93 \%$ reported binge drinking and 65\% an alcohol related harm in the past year. Most players would turn to family (36\%) or friends (21\%) for help. There were significant associations between monthly bingeing (OR 18.4), smoking (OR 3.3), generally drinking in public (OR 3.2), current gambling (OR 2.3), male gender (OR 2.1), an alcohol harm in the past year (OR 1.9) and adverse alcohol use. In contrast, co-habiting with a partner (OR 0.5) was protective.

Conclusions Excess alcohol consumption, alcohol related harms and binge drinking are prevalent in an elite sporting population, particularly during the off-season. Specific strategies are required to reduce alcohol related harms, particularly amongst high-risk groups during the off-season.
\end{abstract}

Keywords Addiction $\cdot$ Alcohol $\cdot$ Athlete $\cdot$ Dependence

\section{Introduction}

Alcohol is the seventh leading risk factor for death worldwide, causing $6.8 \%$ of age-standardised male deaths and $2.2 \%$ of age-standardised female deaths [1]. The impact of

Kieran Murray

kemurray@hotmail.com

1 University Hospital Limerick, Dooradoyle, Limerick, Ireland

2 Washington Street Medical Centre, Cork, Ireland

3 Department of Public Health, HSE Midlands, Tullamore, Offaly, Ireland

4 Children's Health Ireland, Dublin 1, Ireland

5 School of Public Health, University College Dublin, Dublin 4, Ireland

6 Beaumont Hospital and RCSI, Dublin 9, Ireland

7 Alcohol Health Alliance Ireland, Dublin 1, Ireland alcohol on mortality is highest in the $25-49$ age group, in contrast to many other conditions, and makes evaluation of alcohol consumption patterns in elite athletes important. Annual pure alcohol consumption is 9.71 year in the UK and 11 1/year in Ireland [2]. In the UK, alcohol causes 31,000 deaths and consumes $3.2 \%$ of health expenditure [3]. In Ireland, alcohol causes 2700 deaths annually and consumes $10 \%$ of health expenditure [4]. Alcohol consumption in Ireland has increased during the COVID-19 pandemic [5]; 76\% of the Irish population are current drinkers, of whom 39\% typically binge drink ( $\geq 6$ standard drinks in one sitting) [6]. Binge drinking rates are higher in 15-24-year-olds (48\%) and in 25-34-year-olds (55\%) in Ireland [6]. Binge drinkers have higher all-cause mortality than steady drinkers [7].

Alcohol is deeply intertwined with sport, despite the evidence that alcohol impairs sporting performance by decreasing skill levels and aerobic capacity $[8,9]$. Athletes are more prone to binge drink than non-athletic peers [10-12]. Team members can 
feel obliged to drink to increase team bonding [9]. Hazing can include forced binge drinking for new players which is clearly potentially harmful $[13,14]$. In a study of US college athletes, $12 \%$ reported forced drinking until vomiting/loss of consciousness [15].

The Gaelic Athletic Association (GAA) is Ireland's largest sporting organisation with over 800,000 members [16]. It is responsible for Gaelic games (Gaelic football, hurling, handball and rounders) [16]. Typically played over a 6-month season, Gaelic football and hurling are amateur, high-intensity, multidirectional contact field sports [17]. These are the most attended field sports in Ireland, with 1.5 million people attending the elite (intercounty) championships annually prior to the COVID-19 pandemic [18]. The Gaelic Players Association (GPA) and Women's Gaelic Players Association (WGPA) are the players' representative bodies with a

Table 1 Characteristics of study participants $(n=717)$

\begin{tabular}{ll}
\hline Male & $535(75 \%)$ \\
Mean (SD) age, years & $24(4)$ \\
Unmarried & $664(93 \%)$ \\
Employment status & \\
Full-time employment & $384(54 \%)$ \\
Student & $302(42 \%)$ \\
Part-time employed & $25(4 \%)$ \\
Unemployed & $5(1 \%)$ \\
Educational status & \\
Completed university & $477(67 \%)$ \\
Completed secondary & $235(33 \%)$ \\
Completed primary only & $5(1 \%)$ \\
Smoking status & \\
Non-smoker (past year) & $551(77 \%)$ \\
Less than daily & $156(22 \%)$ \\
Daily & $10(1 \%)$ \\
Living arrangement & \\
Parents & $395(55 \%)$ \\
Partner & $135(19 \%)$ \\
Intercounty teammates & $47(7 \%)$ \\
Other friends & $119(17 \%)$ \\
Other & $21(3 \%)$ \\
Playing status & \\
Playing & $256(36 \%)$ \\
Injured & $221(31 \%)$ \\
Gports played at elite level & $132(18 \%)$ \\
Hurling & $108(15 \%)$ \\
Both & \\
Playing division & $77(11 \%)$ \\
1 & \\
2 & \\
3 & $373(52 \%)$ \\
4 & $323(45 \%)$ \\
\hline
\end{tabular}

combined membership of around 3500 [19]. A recent survey of elite male GAA players found high levels of adverse alcohol use during the off-season [20]. This report suggested that additional studies were needed to understand the underlying mechanisms driving these patterns of alcohol consumption [20]. Alcohol consumption amongst elite female GAA players has not been examined.

Sports sponsorship is a key element of alcohol marketing with an array of activities used to portray a link between alcohol, sports and elite athletes [21]. In televised English Premiership football, there are on average almost two visual alcohol references per minute [22]. In Ireland, alcohol sponsorship of sport is widespread [rugby (Guinness RBS 6 Nations, Guinness Pro14 League, Heineken Cup) and soccer (Carlsberg's sponsorship of the Football Association of Ireland)]. Guinness previously sponsored the elite GAA hurling national championship.

\section{Methods}

\section{Participants}

All current GPA and WGPA members aged 18 and above were invited to participate. Current elite (senior intercounty) GAA players in Gaelic football/hurling/both were included for analysis. Retired players were excluded.

\section{Design}

This was a point prevalence study to assess alcohol behaviours and culture amongst elite GAA players. The questionnaire was embedded in a larger survey entitled Alcohol and Gambling in Intercounty GAA Players. The incentivised anonymous self-administered electronic SurveyMonkeyTM questionnaire was sent electronically to all GPA and WGPA

Table 2 Alcohol consumption by elite GAA players

\begin{tabular}{ll}
\hline Currently drink alcohol (past year) & $685(96 \%)$ \\
Binge in past year $^{\mathrm{a}}$ & $600(93 \%)$ \\
${\text { Annual intake of pure } \text { alcohol }^{\mathrm{a}}}^{\text {All players }}$ & Median (IQR), litres \\
Male & $2.1(1.1-3.7)$ \\
Female & $2.2(1.3-3.9)^{\mathrm{b}}$ \\
Age at first alcoholic drink, years & $1.6(0.9-3.1)^{\mathrm{b}}$ \\
Mean (SD) & \\
$<16$ & $16.5(2)$ \\
$16-17$ & $155(25 \%)$ \\
$>17$ & $332(54 \%)$ \\
\hline
\end{tabular}

\footnotetext{
${ }^{\mathrm{a}}$ Amongst current drinkers

${ }^{\mathrm{b}}$ Men versus women, $p=0.009$
} 
Table 3 Seasonal variation of alcohol behaviours of elite GAA players

\begin{tabular}{|c|c|c|c|}
\hline & $\begin{array}{l}\text { Off-season } \\
\text { Median (IQR) }\end{array}$ & $\begin{array}{l}\text { In-season } \\
\text { Median (IQR) }\end{array}$ & $p$ value \\
\hline Number of drinking days in a 28 -day period & $3(2-4)$ & $1(1-2)$ & $<0.001$ \\
\hline Standard drinks per drinking day & $7(5-10)$ & $6(3-9)$ & $<0.001$ \\
\hline Standard drinks per 28 days & $20(10-40)$ & $8(4-14)$ & $<0.001$ \\
\hline
\end{tabular}

members aged $\geq 18$ years on 8 th April 2019. The survey was resent once to non-respondents. The average completion time was $9 \mathrm{~min}$.

There were 10 demographic questions (age, gender, education, smoking status, marital status, employment, living arrangements, playing status, sport and playing level). Current drinking status (question 11) was defined as within the last year. If the participant answered 'no', they avoided the questions on alcohol behaviours and skipped to the alcohol culture section. This skip-logic was performed, following feedback from the pilot, to maximise survey integrity and completion rate.

The validated AUDIT-C questionnaire (frequency, quantity of alcohol consumption and frequency of binge drinking) was used to assess adverse alcohol use amongst current drinkers $[23,24]$. A total score from 0 to 12 is obtained by adding the three items outlined above. A score $\geq 5$ indicates adverse alcohol use. The AUDIT-C is the first three questions of the Alcohol Use Disorders Identification Test (AUDIT) created by the WHO in 1992 [25].

Alcohol consumption was assessed using a quantity-frequency approach, as is routine in alcohol surveys [26]. Players were asked about the frequency of alcohol consumption and the number of standard alcoholic drinks consumed, both during and outside of the elite playing season (league and championship). A standard drink was defined as containing 10 grams of alcohol, based on Irish pub measures [27]. This 10 gram measurement is widespread internationally (Spain, Italy, New Zealand and Australia) [28]. Binge drinking rates were also assessed. Prevalence of past-year alcohol-related harms was evaluated using 13 questions validated in a 2002 national study subsequently used in a GAA study [29-31].

Location of drinking, team alcohol policy and age at first alcohol consumption were examined, as these have been predictive of risky drinking in prior studies [31,32]. There were further questions on relative drinking quantities when drinking with or without teammates, location of drinking, team alcohol policy, forced drinking for new players, alcohol culture, promotion and sponsorship. An optional free text section entitled enabled player feedback.

On completion of the survey, relevant alcohol and gambling support groups were provided. To improve the response rate, participants completing the survey were given a code to potentially obtain a voucher (for a sports store or supermarket).

\section{Statistical analysis}

Normal data is presented as mean and standard deviation (SD), and non-normally distributed data are presented as median and interquartile range (IQR). Categorical data are presented as the number (\%). Differences between groups were analysed using unpaired 2-tailed $t$-tests, Mann-Whitney $U$ test, chi-square or Fisher's exact tests as appropriate.

Table 4 Alcohol behaviours and culture in elite GAA players

\begin{tabular}{ll}
$\begin{array}{l}\text { General drink alcohol in a public place (bar/nightclub) } \\
\text { rather than in private }\end{array}$ & $549(95 \%)$ \\
Team alcohol policy & \\
Informal (player-led) & $313(51 \%)$ \\
Individual choice & $206(34 \%)$ \\
Formal policy & $94(15 \%)$ \\
Major influence on team alcohol culture & \\
Players & $309(50 \%)$ \\
External factors (for example Irish society in & $218(36 \%)$ \\
general) & \\
Management & $86(14 \%)$ \\
Alcohol consumption with intercounty teammates & \\
No difference & $317(53 \%)$ \\
More than other nights out & $210(35 \%)$ \\
Less than other nights out & $72(12 \%)$ \\
Forced binge drinking for new players (hazing) & $38(6 \%)$ \\
Feel harmful alcohol culture in GAA & $212(35 \%)$ \\
Received alcohol promotion in the past year & $296(48 \%)$ \\
Approve of alcohol industry sponsorship of GAA & $369(60 \%)$ \\
competitions & \\
Approve of gambling industry to sponsor GAA com- & $166(28 \%)$ \\
petitions & \\
Most likely source of support for addiction issues & \\
Family & $214(36 \%)$ \\
Friends (other than teammate) & $123(21 \%)$ \\
Partner & $92(16 \%)$ \\
GPA/WGPA & $90(15 \%)$ \\
Counsellor & $24(4 \%)$ \\
Intercounty teammates & $24(4 \%)$ \\
Manager/selector & $6(1 \%)$ \\
Team doctor & $4(1 \%)$ \\
Other doctor & $5(1 \%)$ \\
Other & \\
\hline
\end{tabular}

${ }^{\mathrm{a}}$ Gaelic Players Association (GPA) and Women's Gaelic Players Association (WGPA) are the elite players' representative bodies 
Table 5 Alcohol harms in the past year amongst current drinkers

\begin{tabular}{ll}
\hline Regretted something said when drinking & $227(38 \%)$ \\
Physical sickness & $160(27 \%)$ \\
Felt the need to cut down on drinking & $153(26 \%)$ \\
Work/college absence & $148(25 \%)$ \\
Harm to work/studies & $113(19 \%)$ \\
Harm to home life/marriage/relationship & $57(10 \%)$ \\
Harm to health & $49(8 \%)$ \\
Verbally abusive & $43(7 \%)$ \\
Harm to friendship/social life & $36(6 \%)$ \\
Fight & $33(6 \%)$ \\
Accident & $14(2 \%)$ \\
Emergency Department attendance & $13(2 \%)$ \\
Damage to public property & $9(2 \%)$ \\
$\geq 1$ alcohol-related harm & $389(65 \%)$ \\
$\geq 6$ alcohol-related harms & $38(15 \%)$ \\
\hline
\end{tabular}

Univariate analyses were performed for predictors of adverse alcohol use (AUDIT-C score $\geq 5$ ). Variables with $p$ values $<0.2$ in the univariate were then put into a multivariate logistic regression analysis model. Despite not meeting statistical significance in the univariate model, age was retained in the multivariate model as is standard practice in epidemiological analyses to retain this biologically important confounder. Data analyses were performed using SPSS 26.0.

\section{Results}

\section{Participants}

There were 773 respondents. Retired players $(n=54)$ and those who did not clarify playing status $(n=2)$ were excluded; 717 players were included in the study. Participant characteristics are shown in Table 1. The mean age was 24 years. Most respondents were male, unmarried, nonsmokers, in full-time employment, had completed university and were living with their parents. There was a distribution of players across GAA sports and divisions.

\section{Alcohol intake, behaviours and culture}

Reported rates of current alcohol use (96\%) and binge drinking (93\% amongst current drinkers) were high (Table 2). In contrast, total annual alcohol intake was low in both men and women. The mean age at first drink was 16 years old (Table 2); 437 (73\% of current drinkers) exhibited adverse alcohol use (AUDIT-C $\geq 5$ ). The mean AUDIT-C score was
Table 6 Univariate and multivariate analysis for adverse alcohol use (AUDIT-C $\geq 5$ )

\begin{tabular}{|c|c|c|c|}
\hline & $N(\%)$ & $\begin{array}{l}\text { Univariate analysis } \\
\text { OR }(95 \% \mathrm{CI})\end{array}$ & $\begin{array}{l}\text { Multivariate analysis } \\
\text { OR }(95 \% \mathrm{CI})\end{array}$ \\
\hline Male & $454(76 \%)$ & $2.3(1.5-3.5)$ & $2.1(1.2-3.6)$ \\
\hline Age $<25$ years old & $321(54 \%)$ & $1.1(0.7-1.5)$ & $1.6(0.9-2.7)$ \\
\hline Single & $554(92 \%)$ & $1.3(0.7-2.5)$ & \\
\hline Lives with partner & $120(20 \%)$ & $0.6(0.4-0.9)$ & $0.5(0.2-0.9)$ \\
\hline Full-time employed & $332(55 \%)$ & $1.0(0.7-1.5)$ & \\
\hline Student & $243(41 \%)$ & $1.0(0.7-1.4)$ & \\
\hline Completed university & $396(66 \%)$ & $1.0(0.7-1.5)$ & \\
\hline Smoker & $139(23 \%)$ & $4.7(2.6-8.5)$ & $3.3(1.5-7.2)$ \\
\hline Elite (division 1) & $206(34 \%)$ & $1.3(0.9-1.8)$ & \\
\hline Footballer only & $317(53 \%)$ & $0.7(0.5-1.0)$ & $0.7(0.5-1.2)$ \\
\hline Injured (versus playing) & $64(11 \%)$ & $0.8(0.5-1.4)$ & \\
\hline Monthly binger & $254(42 \%)$ & $25.0(12.0-52.1)$ & $18.4(8.5-40.0)$ \\
\hline An alcohol harm in past year & $388(65 \%)$ & $3.4(2.3-4.9)$ & $1.9(1.2-3.1)$ \\
\hline Believe harmful alcohol culture within the GAA & $212(35 \%)$ & $1.2(0.8-1.7)$ & \\
\hline $\begin{array}{l}\text { Approve of alcohol industry sponsorship of } \\
\text { GAA competitions }\end{array}$ & $369(60 \%)$ & $0.9(0.6-1.4)$ & \\
\hline Alcohol promotion in the past year & $296(48 \%)$ & $1.3(0.9-1.8)$ & $0.9(0.6-1.5)$ \\
\hline Drink more alcohol with teammates & $210(35 \%)$ & $1.2(0.8-1.8)$ & \\
\hline Formal team alcohol policy & $94(15 \%)$ & $0.7(0.4-1.1)$ & $0.6(0.3-1.1)$ \\
\hline Players most influential on alcohol culture & $309(50 \%)$ & $1.2(0.8-1.7)$ & \\
\hline Binge drinking as an initiation to a team & $38(6 \%)$ & $1.3(0.6-2.8)$ & \\
\hline 18 or more at first alcoholic drink & $124(20 \%)$ & $0.5(0.3-0.7)$ & $0.8(0.5-1.4)$ \\
\hline Generally drink in public & $549(95 \%)$ & $3.6(1.7-7.4)$ & $3.2(1.2-8.5)$ \\
\hline Gambled in past year & $479(79 \%)$ & $3.5(2.3-5.4)$ & $2.3(1.3-3.9)$ \\
\hline
\end{tabular}


5.5 (SD 1.9). The mean AUDIT-C was higher in males at 5.7 (SD 1.8) than in females at 5.0 (SD 2) $(p<0.001)$. Alcohol intake was higher in the off-season (Table 3 ).

Alcohol behaviours and culture are outlined in Table 4; $95 \%$ of players generally drank alcohol in a public setting (bar/nightclub) as opposed to a private setting (home). Roughly half of the teams had a player-led alcohol policy, whilst $15 \%$ had a formal policy from management. There were very low levels of forced binge drinking and hazing for new players. Most players would turn to family (36\%) or friends (21\%) for help with addiction issues (Table 4); $48 \%$ of players had received alcohol promotion in the past year.

$65 \%$ of current drinkers reported alcohol-related harm in the past year and $15 \%$ had $\geq 6$ alcohol-related harms in the past year. The most common alcohol-related harm was regretting something said when drinking (32\%); 148 (25\%) reported absence from work or college and $33(5 \%)$ a physical fight; 57 (10\%) reported harm to home life/marriage/ relationship, and 13 (2\%) required Emergency Department attendance (Table 5).

\section{Predictors of adverse alcohol use}

Univariate and multivariate analyses for predictors of adverse alcohol use are shown in Table 6. Monthly binge drinking (OR 18.4), smoking (OR 3.3), generally drinking in public (OR 3.2), current gambling (OR 2.3), male gender (OR 2.1) and a past-year alcohol harm (OR 1.9) had statistically significant associations with adverse alcohol use in multivariate analysis (Table 6). In contrast, cohabiting with a partner (OR 0.5) appeared protective (Table 6). Nagelkerke $R$ square value for the multivariate prediction model was 0.454 .

\section{Conclusions}

\section{Main findings}

These data demonstrate concerning alcohol behaviours amongst elite athletes of both genders. The results of our study demonstrate high levels of alcohol-related harms and binge drinking. Our findings replicate and expand on the results of prior studies of both elite and non-elite male GAA players [18, 20, 31, 33]. Similar to other elite sportspeople, alcohol harm levels in elite GAA players were above the reported populational baseline [12, 31, 32]. 96\% of players currently drink alcohol. This is far higher than the national Irish rate of $76 \%$ [6]. In our study, $73 \%$ of current drinkers displayed adverse alcohol use. Of concern, $35 \%$ drank more with teammates, with only $12 \%$ drinking less; $65 \%$ of current drinkers had alcohol-related harm in the past year, and 2\% required Emergency Department attendance. Alcohol consumption was seasonal with more binge drinking and higher quantities and frequencies of alcohol consumption in the off-season, a pattern seen in elite Australian rules football players [32]. Most players would turn to family (36\%) or friends $(21 \%)$ for help with addiction issues. In multivariate analysis, monthly binge drinking, smoking, generally drinking in public, current gambling, male gender, past-year alcohol harm and not cohabiting with a partner were associated with adverse alcohol use. This is important and useful information. In an era of limited resources, this enables targeted interventions for those most likely to benefit.

The seasonal pattern of alcohol consumption was captured in the players' comments. Many players feel unable to drink alcohol for long periods for numerous reasons (drinking bans, peer pressure, inability to perform in their chosen sport) and then drink heavily when the opportunity arises, as was outlined in respondent comments: there seems to be a culture of full duck or no dinner and 'players tend to abstain from alcohol for long periods of time and then when they do drink they binge heavily. I am definitely guilty of this. The culture in elite GAA appears of drinking to excess as opposed to in moderation. Some respondents suggested that they feel unable to drink in moderation throughout the year and over-indulge in the off-season: 'The extremes are the issue - going from drinking nothing to binge drinking. A culture whereby it was acceptable to have one drink infrequently eg. with dinner, rather than complete enforced abstinence may reduce the extremes'. During the season, players spend long periods together and often have limited social outlets. At the conclusion of competitions, alcohol seems to be an integral part of a celebration of victory or commiseration of defeat. 'Post League or Championship, players on all panels I'd say would do a bender in a pub somewhere due to the fact they haven't drunk in so long and justify it in their own minds that it's ok due to the sacrifice they made of staying off it for months or weeks - whereas if the culture was changed, few drinks after games is fine and players could relax then this wouldn't happen.' Some respondents feel that underage GAA members grow up associating sports with alcohol: '... underage members of our club growing up in the same drinking culture and heavily associating it with GAA. There are also many occurrences of underage drinking as a result. I have seen children as young as 11 or 12 drinking after county final celebrations'.

However, there were also encouraging findings. Annual alcohol consumption amongst players was below national levels. Forced binge drinking as a form of initiation for new teammates was much less prevalent than in North American collegiate sports. Some respondents believe the alcohol culture is changing for the better: 'I do not know if there is a wider problem but within my own camp there does not 
appear to be the same level of consumption or frequency of consumption as there was 10 years ago'.

\section{Methodological considerations}

This study provides a detailed insight into alcohol culture in elite athletes. It describes a large cohort $(n=717)$, including females who are often neglected in studies examining addiction in athletes. All registered active adult players were contacted, ensuring no bias. This study used validated tools, allowing comparison with national data and prior studies. It also offers a detailed examination of alcohol culture. Seasonality of drinking, hazing, attitudes to alcohol sponsorship and sources of support were explored. Understanding these details is key in designing effective interventions to target this high-risk group [18, 31, 33].

The use of self-reported outcome measures is a limitation. Recollection of unpleasant situations can be influenced by denial and repression [34]. However, self-report surveys are often used to assess alcohol use and have been validated $[35,36]$.

\section{Practical implications}

Reversing the harmful drinking culture discussed likely requires a multifaceted approach involving the players, representative bodies, backroom staff, government and GAA policy and wider society.

On a national level, reducing alcohol affordability through taxation or price regulation is the most effective and costeffective way to reduce alcohol-related harms [37]. However, in elite GAA, the players themselves would likely need to be key drivers of change. Over half of respondents reported a player-led alcohol policy, and that players had the most influence on alcohol culture. Improving health behaviours in sportspeople is particularly important to society, as athletes can function as role models, particularly to young people [38-41]. Professional footballer Marcus Rashford's successful campaign to extend means-tested free school meals in the UK over the summer of 2020 provided a clear example of the importance of sportspeople in our societies [42]. Elite athletes have an extended reach. From a public health perspective, influencing those who influence others may prove an effective strategy. Rashford has over 14 million followers on social media (Twitter and Instagram).

There are numerous evidence-based solutions to reduce adverse alcohol use. Sponsorship of sportspeople by the alcohol industry, especially the provision of discounted or free alcoholic beverages, is associated with hazardous drinking [43]. Almost half the respondents in this study have received some form of alcohol promotion in the past year. Previously, the alcohol industry was the main national sponsor of the senior GAA hurling national championship.
In contrast to many other sporting organisations, the GAA is a community-based volunteer organisation that describes player welfare as one of its core values [44]. Interestingly, in 2018 , the GAA prohibited betting firms sponsoring any competition, team, playing gear or facility [45]. This contrasts starkly with many other sporting organisations. In 2017, half of English Premier League teams had a gambling company as shirt sponsor [46]. There is currently a campaign by Alcohol Action Ireland to ban alcohol sponsorship of sport in Ireland to 'decelerate the relentless promotion of alcohol' [21]. The authors believe that the GAA, in the public interest, should continue to show leadership by extending its ban on gambling sponsorship to include the alcohol industry.

Recent years have seen strategies to curb problem alcohol use in Ireland. The GAA and the Health Service Executive launched The Alcohol \& Substance Abuse Prevention (ASAP) Programme and the 'Drink Less and Gain More' campaign [47, 48]. The 2018 Public Health (Alcohol) Act legislated for minimum unit pricing in Ireland, an intervention proven to decrease alcohol consumption [49]. The GPA launched a counselling service to deal with issues such as alcohol addiction. This current study highlights players at high risk of adverse alcohol use (including males, smokers, current gamblers and those not living with a partner).

Screening and brief interventions are beneficial for individuals with addiction issues [50-53]. These have demonstrated health and fiscal improvements for both harmful (damaging health) and hazardous (at risk of an alcoholrelated problem) drinkers [54]. Interventions have successfully reduced risky alcohol consumption amongst athletes $[55,56]$. Perhaps elements of these could be successfully replicated in other sporting organisations. Over two-thirds of players in this study would turn to turn to family, friends or partners for help with addiction issues. This highlights the need to involve these stakeholders, as this is to whom the players are most likely to turn to for support rather than representative bodies, teammates, counsellors, doctors or management.

Limiting alcohol accessibility for underage drinkers is important. The age of the first alcoholic drink has been shown to predict future alcohol consumption [57]. In a prior study of GAA players, age at first drink predicted regular binge drinking [31]. In our study, the mean age of first drink was 16 years old which is below the legal age of alcohol consumption (18 years).

Another potential intervention is to increase taxation on alcohol. More expensive alcohol is particularly effective in decreasing intake in younger, regular and heavy drinkers [58]. The introduction of hypothecated taxes on tobacco can decrease the tobacco industry's funding of sport [59]. This approach could potentially decrease alcohol sponsorship also [59]. The Alcohol Harm Paradox alludes to the finding that lower socioeconomic status groups suffer more 
alcohol-related problems despite drinking less alcohol [60]. The paradox is particularly significant for males and those in younger age groups [60].

\section{Summary}

Overall, these data suggest that, similar to other sportspeople, Gaelic athletes of both genders show high rates of binge drinking and adverse alcohol use with a seasonal drinking pattern occurring mainly outside of the playing season; $65 \%$ of current drinkers had alcohol-related harm in the past year. Worryingly, $35 \%$ of players drank more with teammates, with only $12 \%$ drinking less. Players who are male, smoke, binge drink, generally drink in public, gamble and do not live with a partner appear at particular risk and may benefit from targeted interventions.

Acknowledgements The Gaelic Players Association (GPA) and Women's Gaelic Players Association (WGPA) for cooperation and distribution of the survey.

Author contribution KM conceptually designed the study and was involved in data entry, data analysis and initial drafting of the manuscript. CM was involved in conceptual design, data entry and initial drafting of the manuscript. AH was involved in data analysis and initial drafting of the manuscript. JM was involved in drafting of the manuscript. MC was involved in data analysis and initial drafting of the manuscript. FM was involved in study design and drafting of the manuscript. All authors approved the manuscript. The corresponding author had final responsibility for the decision to submit for publication.

Availability of data and material The dataset used and analysed during the current study is available from the corresponding author on reasonable request.

\section{Declarations}

Ethics approval Ethical approval was provided by the Clinical Research Ethics Committee of the Cork Teaching Hospitals.

Competing interests JM was an elite Gaelic footballer. FM is a chair of the Alcohol Health Alliance Ireland.

\section{References}

1. Griswold MG, Fullman N, Hawley C et al (2018) Alcohol use and burden for 195 countries and territories, 1990-2016: a systematic analysis for the Global Burden of Disease Study 2016. Lancet 392(10152):1015-1035

2. (OECD) OfEC-oaD. Alcohol consumption (2018)[cited 2019. Available from: https://data.oecd.org/healthrisk/alcohol-consumption.htm accessed 27th February 2019.

3. Balakrishnan R, Allender S, Scarborough P et al (2009) The burden of alcohol-related ill health in the United Kingdom. J Public Health 31(3):366-373. https://doi.org/10.1093/pubmed/fdp051

4. Mongan D (2016) Overview of alcohol consumption, alcoholrelated harm and alcohol policy in Ireland. Drugnet Ireland 1-3
5. Central Statistics Office (2020) Social Impact of COVID-19 Survey April 2020. [cited 2020 28th May]. Available from: https://www. cso.ie/en/releasesandpublications/ep/p-sic19/socialimpactofcovid19surveyapril2020/introductionandsummaryofresults/ accessed 26th May 2020

6. Ipsos M (2017) Healthy Ireland 2017: summary of findings. Government Publications

7. Murray RP, Connett JE, Mäkelä P et al (2005) Difficulty in demonstrating a risk from drinking pattern in fourteen years of coronary heart disease morbidity and mortality: the Lung Health Study. Addict Behav 30(5):875-887

8. O'Brien CP (1993) Alcohol and sport. Sports Med 15(2):71-77

9. Maughan RJ (2006) Alcohol and football. J Sports Sci 24(07):741-748

10. Wechsler H, Davenport AE, Dowdall GW et al (1997) Binge drinking, tobacco, and illicit drug use and involvement in college athletics: a survey of students at 140 American colleges. J Am Coll Health 45(5):195-200

11. Leichliter JS, Meilman PW, Presley CA et al (1998) Alcohol use and related consequences among students with varying levels of involvement in college athletics. J Am Coll Health 46(6):257-262

12. O'Brien KS, Blackie JM, Hunter JA (2005) Hazardous drinking in elite New Zealand sportspeople. Alcohol Alcohol 40(3):239-241

13. Diamond AB, Callahan ST, Chain KF et al (2016) Qualitative review of hazing in collegiate and school sports: consequences from a lack of culture, knowledge and responsiveness. Br J Sports Med 50(3):149-153

14. Gladden RM (2014) Bullying surveillance among youths: uniform definitions for public health and recommended data elements. Version 1.0. Centers for Disease Control and Prevention

15. Allan EJ (2009) Hazing in view: college students at risk: initial findings from the National Study of Student Hazing: Diane Publishing

16. Gaelic Athletic Association [cited 2019 27th February]. Available from: https://www.gaa.ie/the-gaa/about-the-gaa/

17. McIntyre M (2005) A comparison of the physiological profiles of elite Gaelic footballers, hurlers, and soccer players. Br J Sports Med 39(7):437-439

18. Gouttebarge V, Tol JL, Kerkhoffs GM (2016) Epidemiology of symptoms of common mental disorders among elite Gaelic athletes: a prospective cohort study. Physician Sportsmed 44(3):283-289

19. Elish Kelly JB, Seamus McGuinness, Dorothy Watson (2018) Playing senior inter-county gaelic games: experiences, realities and consequences. [cited 2019 1st March]. Available from: https:// www.gaelicplayers.com/Portals/0/Publications/RS76.pdf

20. Kelly E, Keegan C, Walsh B (2019) Safeguarding amateur athletes: an examination of player welfare among senior inter-county Gaelic players. ESRI Research Series

21. Ireland AA (2014) Submission to the working group on regulating sponsorship by alcohol companies of a major sporting event

22. Graham A, Adams J (2013) Alcohol marketing in televised English professional football: a frequency analysis. Alcohol Alcohol 49(3):343-348. https://doi.org/10.1093/alcalc/agt140

23. Dawson DA, Grant BF, Stinson FS et al (2005) Effectiveness of the derived Alcohol Use Disorders Identification Test (AUDIT-C) in screening for alcohol use disorders and risk drinking in the US general population. Alcohol Clin Exp Res 29(5):844-54

24. de Meneses-Gaya C, Zuardi AW, Loureiro SR et al (2009) Alcohol Use Disorders Identification Test (AUDIT): an updated systematic review of psychometric properties. Psychology \& Neuroscience 2(1):83

25. Saunders JB, Aasland OG, Babor TF et al (1993) Development of the alcohol use disorders identification test (AUDIT): WHO collaborative project on early detection of persons with harmful alcohol consumption-II. Addiction 88(6):791-804 
26. Greenfield TK (2000) Ways of measuring drinking patterns and the difference they make: experience with graduated frequencies. J Subst Abuse 12(1-2):33-49

27. Hope A (2020) Typical drinks in Ireland. Department of Health and Children

28. Health N, Council MR (2007) Australian alcohol guidelines for low-risk drinking. Draft for public consultation, Australian Capital Territory Canberra

29. Ramstedt M, Hope A (2005) The Irish drinking habits of 2002drinking and drinking-related harm in a European comparative perspective. J Subst Use 10(5):273-283

30. Dawson DA, Room R (2000) Towards agreement on ways to measure and report drinking patterns and alcohol-related problems in adult general population surveys: the Skarpö Conference overview: Elsevier

31. O'Farrell AM, Allwright SP, Kenny SC et al (2010) Alcohol use among amateur sportsmen in Ireland. BMC Res Notes 3(1):313

32. Dietze PM, Fitzgerald JL, Jenkinson RA (2008) Drinking by professional Australian Football League (AFL) players: prevalence and correlates of risk. Med J Aust 189(9):479-483

33. O'Farrell A, Kingsland M, Kenny S et al (2018) A multi-faceted intervention to reduce alcohol misuse and harm amongst sports people in Ireland: a controlled trial. Drug Alcohol Rev 37(1):14-22

34. Jacobson E (1957) Denial and repression. J Am Psychoanal Assoc $5(1): 61-92$

35. Cooper AM, Sobell MB, Sobell LC et al (1981) Validity of alcoholics' self-reports: duration data. Int J Adhes 16(3):401-406

36. Midanik LT (1988) Validity of self-reported alcohol use: a literature review and assessment. Br J Addict 83(9):1019-1029

37. Burton R, Henn C, Lavoie D et al (2017) A rapid evidence review of the effectiveness and cost-effectiveness of alcohol control policies: an English perspective. Lancet 389(10078):1558-1580

38. Bandura A, Walters RH (1977) Social learning theory: Prenticehall Englewood Cliffs NJ

39. Armour K, Duncombe R (2012) Changing lives? Critical evaluation of a school-based athlete role model intervention. Sport Educ Soc 17(3):381-403

40. Payne W, Reynolds M, Brown S et al (2003) Sports role models and their impact on participation in physical activity: a literature review. Victoria: VicHealth 74

41. Bush AJ, Martin CA, Bush VD (2004) Sports celebrity influence on the behavioral intentions of generation Y. J Advert Res 44(1):108-118

42. Sinha IP, Lee AR, Bennett D et al (2020) Child poverty, food insecurity, and respiratory health during the COVID-19 pandemic. Lancet Respir Med 8(8):762-763

43. O’Brien KS, Kypri K (2008) Alcohol industry sponsorship and hazardous drinking among sportspeople. Addiction 103(12):1961-1966

44. Gaelic Athletic Association (GAA) mission, vision and values https://www.gaa.ie/the-gaa/administration/: Gaelic Athletic Association; [cited 2019 29th April]. Available from: https://www.gaa. ie/the-gaa/administration/ accessed 29th April 2019.

45. Gaelic Athletic Association (2018) GAA Launch Gambling Awareness Campaign Reduce the Odds [updated 9 August 2018; cited 2019 5th March]. Available from: https://www.gaa.ie/news/ gaa-launch-gambling-awareness-campaign-reduce-the-odds/ accessed 5th March 2019
46. Lopez-Gonzalez H, Griffiths MD (2017) Betting, forex trading, and fantasy gaming sponsorships - a responsible marketing inquiry into the 'Gamblification'of English football. Int J Ment Health Addict 1-16

47. Gaelic Athletic Association (2016) The GAA ASAP Programme: Gaelic Athletic Association; 2016 [cited 2019 5th March]. Available from: https://www.gaa.ie/news/the-gaa-asap-programme/ accessed 5th March 2019

48. Gaelic Athletic Association (2018) GAA and HSE announce Drink Less and Gain More campaign [updated 31st July 2018; cited 2019 30th April]. Available from: https://www.gaa.ie/news/ gaa-and-hse-announce-drink-less-and-gain-more-campaign/ accessed 30th April 2019

49. Stockwell T, Auld MC, Zhao J et al (2012) Does minimum pricing reduce alcohol consumption? The experience of a Canadian province. Addiction 107(5):912-920

50. Bien TH, Miller WR, Tonigan JS (1993) Brief interventions for alcohol problems: a review. Addiction 88(3):315-336

51. Petry NM, Weinstock J, Ledgerwood DM et al (2008) A randomized trial of brief interventions for problem and pathological gamblers. J Consult Clin Psychol 76(2):318

52. Hodgins DC, Currie S, el-Guebaly N et al (2004) Brief motivational treatment for problem gambling: a 24-month followup. Psychol Addict Behav 18(3):293

53. Kaner EFS, Beyer FR, Muirhead C et al (2018) Effectiveness of brief alcohol interventions in primary care populations. Cochrane Database Syst Rev (2). https://doi.org/10.1002/ 14651858.CD004148.pub4

54. Skog OJ (1985) The collectivity of drinking cultures: a theory of the distribution of alcohol consumption. Br J Addict 80(1):83-99

55. Perkins HW, Craig DW (2006) A successful social norms campaign to reduce alcohol misuse among college student-athletes. J Stud Alcohol 67(6):880-889

56. Kingsland M, Wolfenden L, Tindall J et al (2015) Tackling risky alcohol consumption in sport: a cluster randomised controlled trial of an alcohol management intervention with community football clubs. J Epidemiol Community Health 69(10):993-999

57. Pedersen W, Skrondal A (1998) Alcohol consumption debut: predictors and consequences. J Stud Alcohol 59(1):32-42

58. Chaloupka FJ, Wechsler H (1996) Binge drinking in college: the impact of price, availability, and alcohol control policies. Contemp Econ Policy 14(4):112-124

59. O'Brien KS, Miller PG, Webber A et al (2011) Alcohol industry and non-alcohol industry sponsorship of sportspeople and drinking. Alcohol Alcohol 46(2):210-213. https://doi.org/10.1093/ alcalc/agq095

60. Beard E, Brown J, West R et al (2016) Deconstructing the alcohol harm paradox: a population based survey of adults in England. PloS one 11(9):e0160666

Publisher's Note Springer Nature remains neutral with regard to jurisdictional claims in published maps and institutional affiliations. 